\title{
Pharmacological targeting of guanosine monophosphate synthase suppresses melanoma cell invasion and tumorigenicity
}

\author{
A Bianchi-Smiraglia', JA Wawrzyniak ${ }^{1}$, A Bagati ${ }^{1}$, EK Marvin ${ }^{1}$, J Ackroyd ${ }^{2}$, S Moparthy ${ }^{1}$, W Bshara ${ }^{3}$, EE Fink ${ }^{1}$, CE Foley ${ }^{1}$, GE Morozevich ${ }^{4}$, \\ AE Berman ${ }^{4}$, DS Shewach ${ }^{2}$ and MA Nikiforov ${ }^{*}, 1$
}

Malignant melanoma possesses one of the highest metastatic potentials among human cancers. Acquisition of invasive phenotypes is a prerequisite for melanoma metastases. Elucidation of the molecular mechanisms underlying melanoma invasion will greatly enhance the design of novel agents for melanoma therapeutic intervention. Here, we report that guanosine monophosphate synthase (GMPS), an enzyme required for the de novo biosynthesis of GMP, has a major role in invasion and tumorigenicity of cells derived from either BRAF ${ }^{\mathrm{V} 600 \mathrm{E}}$ or NRAS ${ }^{\mathrm{Q61R}}$ human metastatic melanomas. Moreover, GMPS levels are increased in metastatic human melanoma specimens compared with primary melanomas arguing that GMPS is an attractive candidate for anti-melanoma therapy. Accordingly, for the first time we demonstrate that angustmycin A, a nucleoside-analog inhibitor of GMPS produced by Streptomyces hygroscopius efficiently suppresses melanoma cell invasion in vitro and tumorigenicity in immunocompromised mice. Our data identify GMPS as a powerful driver of melanoma cell invasion and warrant further investigation of angustmycin $A$ as a novel anti-melanoma agent.

Cell Death and Differentiation (2015) 22, 1858-1864; doi:10.1038/cdd.2015.47; published online 24 April 2015

Malignant melanoma is one of the most aggressive types of human cancers. Its ability to metastasize in combination with resistance to conventional anticancer chemotherapy makes melanoma extremely difficult to cure, and the median survival of patients with metastatic melanoma is 8.5 months. $^{1-3}$ A better understanding of the biology behind melanoma aggressiveness is imperative to facilitate the development of novel anti-melanoma strategies.

Melanoma and other cancers cells have been shown to strongly rely on de novo nucleotide biosynthesis ${ }^{4,5}$ and often overexpress several biosynthetic enzymes involved in these pathways. ${ }^{6-9}$ Recently, we have identified a fundamental connection between melanoma invasion and biosynthesis of guanylates, ${ }^{8}$ suggesting that distortion of the guanylate metabolism facilitates melanoma progression.

Guanosine monophosphate reductase (GMPR) reduces GMP to one of its precursors, inosine monophosphate (IMP), and depletes intracellular GTP pools (Figure 1a). We have recently demonstrated that GMPR suppresses melanoma cell invasion and growth of human melanoma cell xenografts. These findings tightly linked guanylate production to the invasive potential of melanoma cells. ${ }^{8}$

Of the several enzymes involved in guanylate biosynthesis, inositol monophosphate dehydrogenases 1 and 2 (IMPDH-1, -2), functional antagonists of GMPR (Figure 1a), have been targeted clinically with several drugs including the most specific one, mycophenolic acid (MPA) and its salt, mycophenolate mofetil (MMF). ${ }^{10-13}$ Nonetheless, prior studies demonstrated that MPA possesses poor anti-tumor activity, ${ }^{14,15}$ and it is primarily used as an immunosuppressing agent in organ transplantation. ${ }^{10-12}$

GMP synthase (GMPS) is the other functional antagonist of GMPR. GMPS catalyzes the amination of xanitol monophosphate (XMP) to GMP to promote GTP synthesis (Figure 1a). ${ }^{16,17}$ Most of the studies on GMPS have been performed in bacteria, yeast, and insects, where GMPS has been shown to have a key role in sporulation, pathogenicity, and axon guidance, respectively. ${ }^{18-20}$ Mammalian GMPS has been the subject of several studies addressing its unconventional (GMP-unrelated) roles in the regulation of activity of ubiquitin-specific protease 7 (USP7). ${ }^{21-24}$ However, because of the newly revealed importance of guanylate metabolism in control of melanoma cell invasion and tumorigenicity, ${ }^{8}$ GMPS emerges as an attractive target for anti-cancer therapy.

In the late 1950s, a specific inhibitor of bacterial GMPS, angustmycin A (also known as decoyinine), has been isolated from Streptomyces hygroscopius as a potential antibiotic with sporulation-inducing activity in Bacillus subtilis. ${ }^{25-29}$ Its antitumor activity has never been experimentally explored. In the current study, we investigated the role of GMPS in regulation of melanoma invasion and tumorigenicity, and explored the possibility of targeting GMPS by angustmycin A as a novel anti-melanoma strategy.

${ }_{1}^{1}$ Department of Cell Stress Biology, Roswell Park Cancer Institute, Buffalo, NY 14263, USA; 'Department of Pharmacology, University of Michigan, Ann Arbor, MI 48109, USA; ${ }^{3}$ Department of Pathology, Roswell Park Cancer Institute, Buffalo, NY 14263, USA and ${ }^{4}$ Orekhovich Institute of Biomedical Chemistry, Moscow 119121, Russia ${ }^{*}$ Corresponding author: MA Nikiforov, Department of Cell Stress Biology, Roswell Park Cancer Institute, BLSC L3-317, Buffalo, NY 14263, USA. Tel: +716 845 3374 ; Fax: +716 845 3944; E-mail: mikhail.nikiforov@ roswellpark.org

Abbreviations: GMPS, guanosine monophosphate synthase; MPA, mycophenolic acid; MMF, mycophenolate mofetil

Received 26.2.15; revised 12.3.15; accepted 16.3.15; Edited by M Blagosklonny; published online 24.4.15 
a

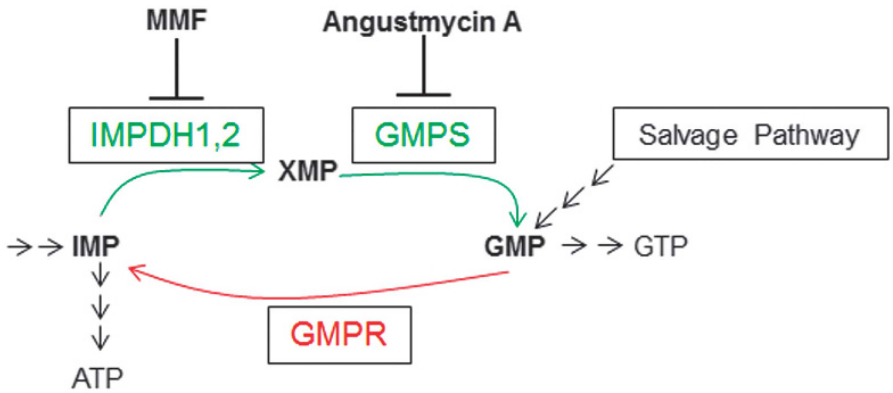

b
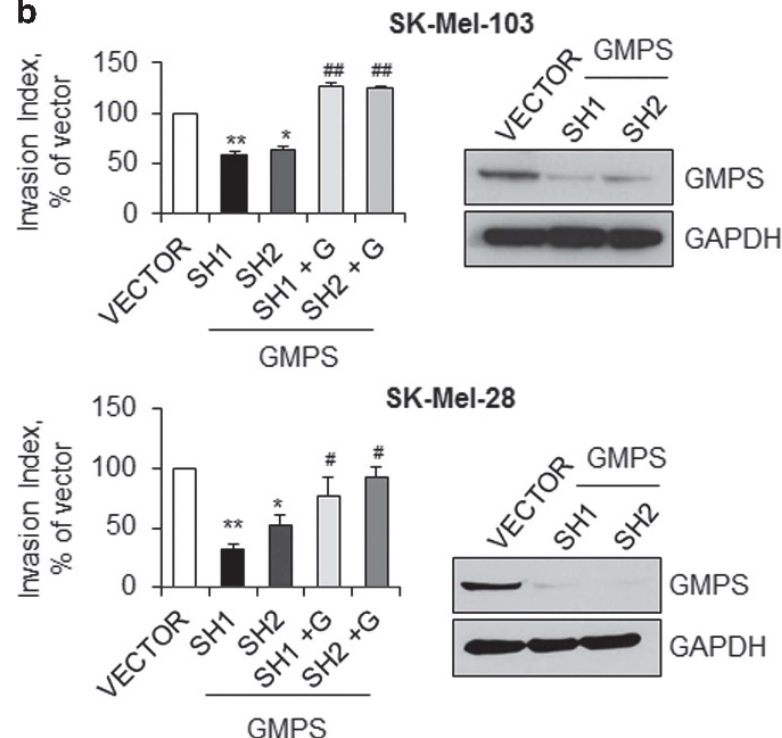

C
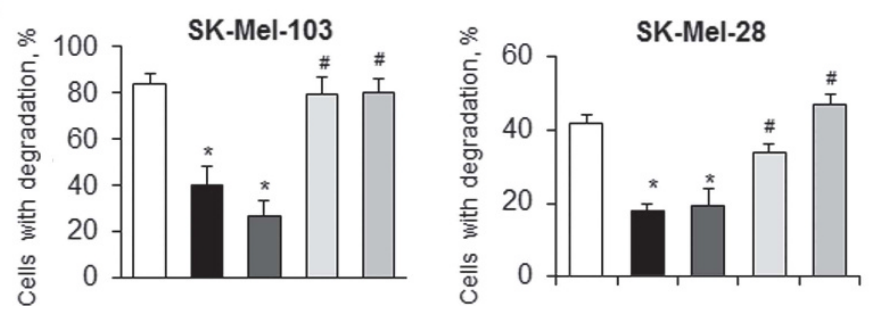

$\square$ VECTOR

- GMPS SH1

- GMPS SH2

$\square$ GMPS $S H 1+G$

$\square$ GMPS SH2+G

Figure 1 GMPS contributes to the invasive capability of melanoma cells. (a) Simplified schematic of the metabolic pathway for guanylates production. (b) SK-Mel-103 and SK-Mel-28 cells were transduced with a control vector or two independent shRNAs to GMPS and tested for invasion through Matrigel (left panel). Where indicated, cells were incubated with $100 \mu \mathrm{M}$ guanosine for $24 \mathrm{~h}$ before the assay and guanosine supplementation was maintained throughout the experimental procedure. The data represent the average \pm S.E.M. of at least two independent experiments. GMPS suppression was verified by immunoblotting (right panel). (c) Cells transduced as in (a) were plated on coverslips coated with FITC-conjugated gelatin. After $16 \mathrm{~h}$ cells were fixed with 4\% PFA and stained for actin (rhodamine-conjugated phallodin) and nuclei (Hoechst). Where indicated, cells were incubated with $100 \mu \mathrm{M}$ guanosine for $24 \mathrm{~h}$ before the assay and guanosine supplementation was maintained throughout the experimental procedure. At least 25 cells/sample were imaged to assess the number of cells with gelatin degradation. The data represent the average \pm S.E.M. of two independent experiments. ${ }^{*} P<0.05$, ${ }^{* *} P<0.001$ compared with control; ${ }^{\#} P<0.05,{ }^{\# \#} P<0.001$ compared with untreated cells. Statistics performed with Student's $t$-Test. See also Supplementary Figure $\mathrm{S} 1$

\section{Results}

GMPS controls melanoma cell invasion. To establish the function of GMPS in melanoma cell invasion, we transduced human metastatic melanoma cells expressing BRAF $^{\mathrm{V} 600 \mathrm{E}}$ (SK-Mel-28) or NRAS ${ }^{\text {Q61R }}$ (SK-Mel-103) with two independent shRNA constructs targeting human GMPS or with a nonsilencing control vector. GMPS depletion was verified by immunoblot (Figure 1b) and cells were tested for the ability to invade through Matrigel in a Boyden chamber assay. We found that GMPS depletion reduced the invasive capability of both cell lines to $\sim 50 \%$ of the vector control (Figure $1 \mathrm{~b}$ ). Most importantly, in both cell lines, supplementation with $100 \mu \mathrm{M}$ guanosine abrogated inhibition of invasion caused by GMPS depletion (Figure 1b). This finding, along with our previous study, ${ }^{8}$ demonstrates that changes in the expression levels of guanylate metabolic enzymes critically affects melanoma cell invasion.

To further evaluate the effects of GMPS depletion on invasion, we assessed the ability of cells transduced with control or GMPS shRNAs to degrade extracellular matrix, a pre-requisite for invasion. We found that GMPS-depleted cells demonstrated significantly reduced ability to degrade 
matrix as compared with control cells. This deficiency was completely annulled by supplementation with guanosine (Figure 1c and Supplementary Figure S1). Therefore, GMPS is essential for full-scale invasion of melanoma cells independently of their BRAF/NRAS statuses.

Depletion of GMPS hinders melanoma cells tumorigenicity. We and others demonstrated that invasion accelerates growth of subcutaneous tumor cell xenografts by relieving the physical constraints of the environment. ${ }^{8,30}$ We therefore were interested in whether GMPS controls tumorigenicity of melanoma cells. To this end, SK-Mel-103 and SK-Mel-28 cells transduced with control vector or GMPS shRNAs were inoculated subcutaneously in both flanks of scid/scid (SCID) mice (5 mice per condition). We defined latency as the time from inoculation at which $50 \%$ of the injected sites had a palpable tumor (SK-Mel-103) or reached $50 \mathrm{~mm}^{3}$ (SK-Mel28). In SK-Mel-103 cells, the latency of GMPS-depleted tumors was 8 days and 15 days (GMPS shRNA-1 and GMPS shRNA-2, respectively) versus 5 days in control cells. SK-Mel-28 cells depleted of GMPS demonstrated tumor latency of 7 days and 10 days (GMPS shRNA-1 and GMPS shRNA-2, respectively) versus 4 days in control cells (Figure 2a). Additionally, GMPS depletion significantly reduced the growth of tumor xenografts derived from both SK-Mel-103 and SK-Mel-28 cells (Figure 2b). These data suggest that GMPS has a prominent role in melanoma tumorigenicity.

GMPS expression is increased in human metastatic specimens. To determine whether GMPS expression is associated with melanoma metastases in patients, we assessed its expression levels via immunohistochemistry in 249 human melanoma specimens that we identified previously. ${ }^{8}$ All patients were divided into one of three cohorts: primary cutaneous thin melanoma (Breslow thickness $\leq 2 \mathrm{~mm}$ ); primary cutaneous thick melanoma (Breslow thickness $>2 \mathrm{~mm}$ ), and metastatic melanoma, consisting of 36, 46, and 167 patients, respectively. Melanoma specimens were scored for intensity of staining and percentage of stained cells as previously described ${ }^{8}$ and the computed IHC index revealed a statistically significant increase in GMPS expression in the metastatic cohort compared with the primary cohorts (Figure 3). No difference was noted within the primary melanoma cohorts between thin and thick melanomas, suggesting that upregulation of GMPS occurs at metastatic stage of melanoma progression. Furthermore, analysis of publically available microarray data generated by 'The Cancer Genome Atlas' (TCGA) Research Network (http://cancergenome.nih.gov/) revealed that GMPS expression is increased in metastatic melanomas compared with primary lesions (Supplementary Figure S2), thus validating the expression pattern of GMPS identified by IHC settings.

Taken together, our data are consistent with the hypothesis that GMPS is a driver of invasive metastatic melanoma and thus may represent a novel anti-melanoma target.

Pharmacological targeting of GMPS by angustmycin A decreases invasion. Angustmycin A is a selective GMPS inhibitor that was tested in mice for non-cancer-related properties. $^{31,32}$ Thus, we were interested in whether GMPS inhibition via angustmycin A may inhibit melanoma cells invasion. Treatment of SK-Mel-28 and SK-Mel103 cells with $2 \mathrm{mM}$ of Angustmycin A (maximum concentration not affecting proliferation of studied cells) reduced their invasion by $\sim 30 \%$ compared with vehicle-treated cells (Figure $4 \mathrm{a}$ ), mirroring the phenotype caused by GMPS depletion (Figure 1b).
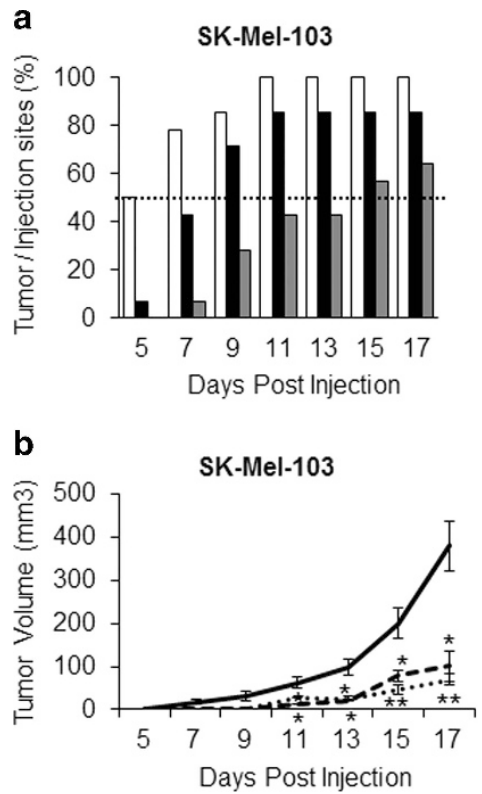
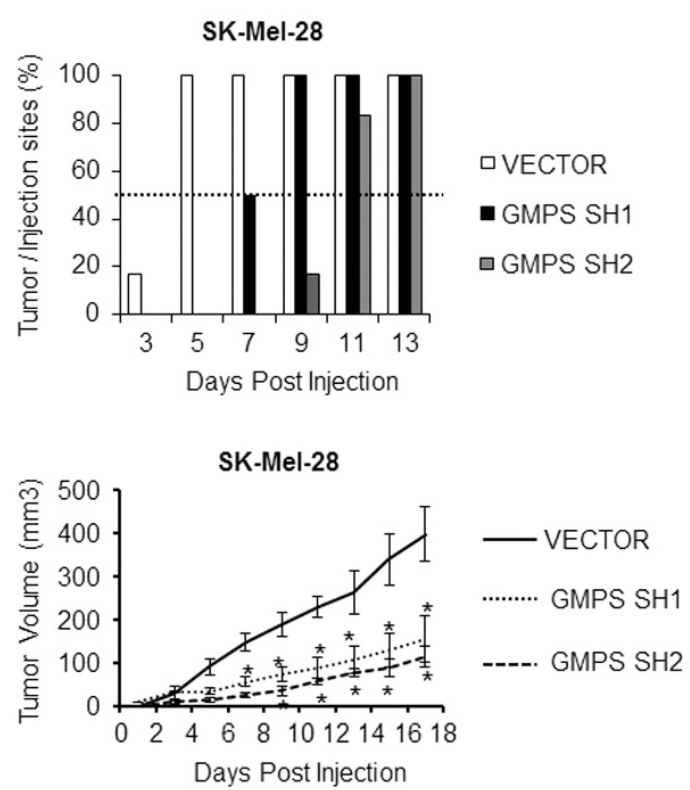

Figure 2 Suppression of GMPS hinders melanoma cell tumorigenicity. (a) SK-Mel-103 and SK-Mel-28 cells were transduced with a control vector or two independent shRNAs to GMPS and inoculated into both flanks of SCID mice ( $n=$ number of inoculation sites). Tumor appearance was recorded when it reached at least 2 mm in one dimension. (b) Tumor xenografts as in (a) were measured every other day. The data represent the average \pm S.E.M. of the tumor size within each cohort. ${ }^{*} P<0.05$; ${ }^{* \star} P<0.001$ by Student's $t$-Test 


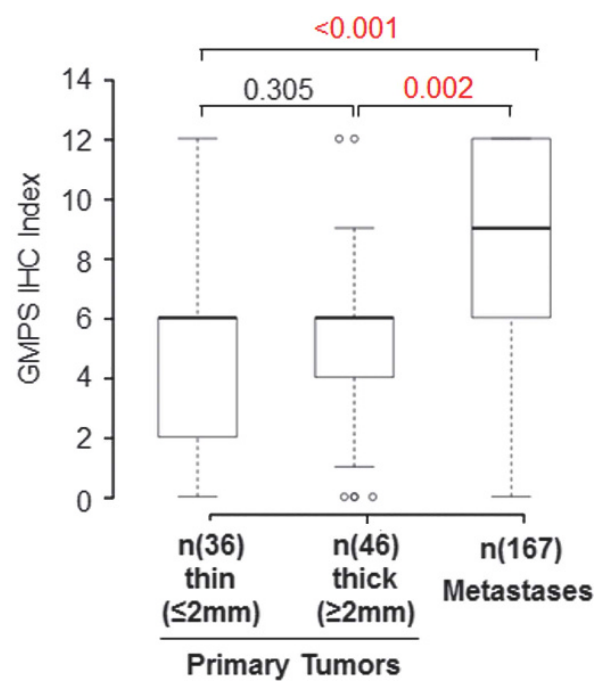

Figure 3 GMPS is upregulated during melanoma progression. Expression of GMPS in thin and thick primary melanomas, and melanoma metastases. The box plots represent the distribution of the $\mathrm{IHC}$ index. The median, first quartile, and third quartile are shown in the 'box' with outlying samples represented by points. The dashed lines represent the interquartile range (IQR) times 1.5 added to the first and third quartiles. The number of patient samples $(n)$ is indicated for each cohort. Statistics were performed with a two-sample $t$-Test. See also Supplementary Figure S2

Furthermore, as observed with GMPS depletion, supplementation with $100 \mu \mathrm{M}$ of guanosine negated the effect of angustmycin A (Figure 4a). Thus, pharmacological inhibition of GMPS phenocopies the effects of GMPS genetic inhibition on melanoma cells invasive ability and its guanosine dependence.

Angustmycin A suppresses growth of melanoma cell xenografts in SCID mice. Based on the above results, we were interested in the anti-melanoma efficacy of angustmycin A in a preclinical mouse model. Previously, the only in vivo study of angustmycin A was performed in mice to investigate immunological responses to skin allografts. ${ }^{31}$ Using data from this paper, we determined the repetitive maximum tolerated dose (rMTD) of angustmycin A in SCID mice as of $120 \mathrm{mg} / \mathrm{kg}$.

IMPDH1 and IMPDH2 are rate-limiting enzymes of de novo guanylate biosynthesis (Figure 1a) and their expression is elevated in tumor cells. ${ }^{7,33,34}$ A specific inhibitor of IMPDH enzymes, MPA has been used as an immunosuppressant during organ transplant. ${ }^{35-37} \mathrm{~A}$ better bioavailable form of MPA is its prodrug MMF (over $200 \%$ improvement in bioavailability), ${ }^{38}$ which has also been proposed as anti-cancer therapy.39,40 Therefore, for the purpose of comparison, we evaluated angustmycin A head-to-head with MMF. Since angustmycin A was delivered i.p. we chose the same route of administration for MMF. We selected a therapeutically active dose of MMF $(30 \mathrm{mg} / \mathrm{kg}$ ) based on previous studies and information on bioavailability. ${ }^{38,40}$

SK-Mel-103 and SK-Mel-28 cells were inoculated subcutaneously in both flanks of SCID mice (18 mice/cell line). Once tumors volume reached approximately $100 \mathrm{~mm}^{3}$, mice were randomly assigned to one of four groups and treated with daily i.p. injections of angustmycin A (120 mg/kg), MMF (30 mg/kg), a

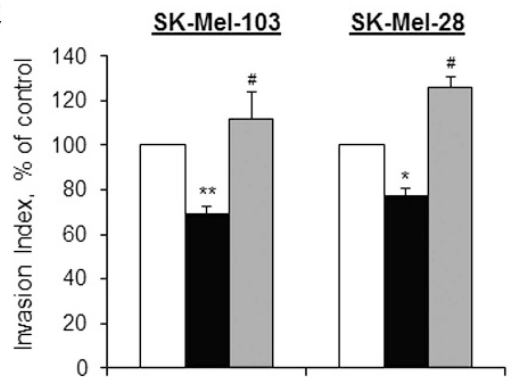

$\square C T R L$ Angustmycin A $\square$ Angustmycin A+Guanosine

b
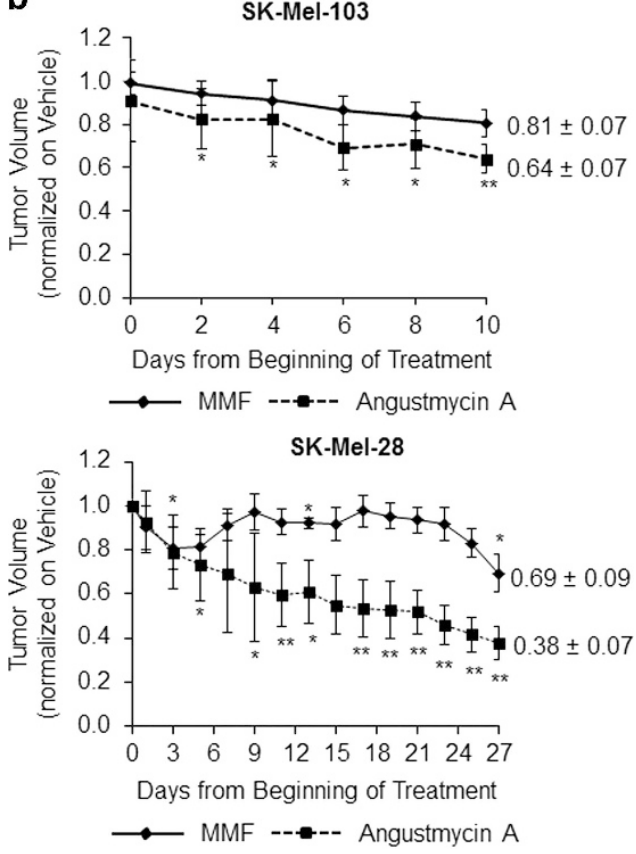

Figure 4 Angustmycin A treatment affects melanoma invasion in vitro and xenograft growth in vivo. (a) SK-Mel-103 and SK-Mel-28 cells were incubated with $2 \mathrm{mM}$ angustmycin $\mathrm{A}$ for $24 \mathrm{~h}$ before invasion assay. Where indicated, cells were supplemented with $100 \mu \mathrm{M}$ guanosine. Control cells were treated with equal volumes of DMSO. Drug treatments were maintained throughout the experimental procedure. (b) SK-Mel-103 and SK-Mel-28 cells were inoculated into both flanks of SCID mice (18 mice/cell line). When tumors reached $100 \mathrm{~mm}^{3}$ in size, mice were randomly assigned to different treatment groups ( 6 mice/condition for the angustmycin A study; 3 mice/condition for the MMF study) and treated with daily i.p. injection of angustmycin A (120 mg/kg in 10\% DMSO in PBS), Mycophenolate Mofetil (MMF, $30 \mathrm{mg} / \mathrm{kg}$ in a suspension of $0.9 \%$ benzyl alcohol, $0.9 \%$ sodium chloride, $0.5 \%$ carboxymethylcellulose, and $0.4 \%$ polysorbate 80 in water), or the correspondent vehicle control. Tumor size was measured every other day. Tumor growth of drugtreated tumors was normalized to the corresponding vehicle control. ${ }^{*} P<0.05$; ${ }^{* *} P<0.001$ compared with vehicle control; ${ }^{\#} P<0.05$ compared to Angustmycin A only treatment by Student's $t$-Test

or with respective vehicles. Tumor size was measured every other day with a caliper and mice were killed once tumor volume reached $1000 \mathrm{~mm}^{3}$ or the animals showed signs of morbidity. In SK-Mel-103 cells, angustmycin A treatment resulted in a $36 \%$ reduction of xenografts volume while MMF caused only $19 \%$ volume reduction compared with respective vehicle controls, (Figure 4b). In SK-Mel-28 cells, the xenograft volume reduction caused by angustmycin $\mathrm{A}$ and MMF were of $62 \%$ and $30 \%$, respectively (Figure $4 b$ ). 


\section{Discussion}

Melanoma is one of the most aggressive types of human cancers, and its lifetime risk and mortality rates have been steadily increasing for decades. ${ }^{41,42}$ In addition, melanomas are exceptionally resistant to chemotherapy treatment and have a high incidence of relapse. ${ }^{43-47}$

Our previous ${ }^{8}$ and current studies have revealed a prominent role of deregulated guanylate pools in melanoma progression. Altered expression of enzymes involved in guanylates biosynthesis priming the cells toward an increase in guanylates production occurs already in primary melanomas (GMPR) and peaks at the metastatic stage (IMPDH2, GMPR, and GMPS) (Wawrzyniak et $a l^{8}$ and Figure 3 and Supplementary Figure S2). Manipulation of the expression levels of these enzymes strongly affects the invasive capacity of melanoma cells and ultimately tumor growth.

The formal possibility exists that the effects seen in the current work are due, at least in part, to GMP biosynthesisunrelated functions of GMPS in regulation of the activity of ubiquitin-specific protease 7 (USP7). ${ }^{21-24}$ However, the ability of exogenous guanosine to fully revert all phenotypes caused by GMPS depletion in vitro (Figure 1) strongly suggests that guanylate biosynthetic function has the most important role in GMPS-dependent maintenance of invasive and tumorigenic phenotypes of melanoma cells.

As of yet, there is no specific therapy against melanoma with NRAS mutations ( $20 \%$ of all melanomas), while melanomas with mutant BRAF ${ }^{\mathrm{V} 600 \mathrm{E}}$ ( $\sim 60 \%$ of all melanomas) are treated with drugs such as vemurafenib and dabrafenib. However, these agents are not effective in $\sim 30 \%$ of patients with BRAF $^{\mathrm{V} 600 \mathrm{E}}$ melanomas and, of the responders, most relapse within $\sim 6$ months. ${ }^{48,49}$

Importantly, we identified GMPS as a positive regulator of invasive and tumorigenic potential of melanoma cells irrespective of their BRAF/NRAS mutation status (Figures 1 and 2). This evidence combined with the finding that GMPS expression is increased in metastatic melanomas (Figure 3, Supplementary Figure S2), makes of GMPS an attractive candidate for therapeutic targeting.

Although the specific GMPS inhibitor, angustmycin A, has been known since the mid-1950s, its antitumor potential has never been thoroughly investigated. Here, we show that angustmycin A treatment affects melanoma invasion in vitro (Figure 4a) and hinders tumor growth in xenograft mouse model (Figure 4b). Moreover, like GMPS activity, the effect of angustmycin $A$ does not appear to be subtype specific as it impaired growth of xenografts from cells harboring NRAS ${ }^{\text {Q61R }}$ (SK-Mel-103) or BRAF ${ }^{\mathrm{V} 600 \mathrm{E}}$ (SK-Mel-28) mutations (Figure 4). So far, angustmycin $A$ has been mainly employed as an antibiotic and most of the studies on its activity have been conducted in bacteria or other lower organisms where it was shown that guanylates have a key role in several aspects of their biology including sporulation, pathogenicity, and axon guidance. ${ }^{18-20,28,29}$ A single study performed in mice failed to reveal any immune-suppressive efficacy, however, it showed good tolerability. ${ }^{31}$

MMF, an inhibitor of IMPDH enzymes, is currently in clinical use as an immunosuppressant although two recent papers suggested that MMF may possess anti-cancer activities in vivo. ${ }^{39,40}$ However, in our head-to-head comparison we demonstrated that the anti-melanoma activity of MMF is significantly lower than that of angustmycin A.

Taken together, our data establish that GMPS levels are increased in human metastatic melanoma specimens and GMPS functions as a driver of both BRAF ${ }^{\mathrm{V} 600 \mathrm{E}}$ and NRAS ${ }^{\mathrm{Q} 61 \mathrm{R}}$ melanoma cell invasion and tumorigenicity. Furthermore, we for the first time demonstrate that pharmaceutical inhibition of GMPS by angustmycin A has the potential to be effective as an anti-melanoma therapy for tumors carrying BRAF ${ }^{\mathrm{V} 600 \mathrm{E}}$ or NRAS ${ }^{\text {Q61R }}$ mutations. Therefore, it would be important to evaluate the potential of angustmycin A as an anti-melanoma drug in clinical settings.

\section{Materials and Methods}

Cell lines. SK-Mel-28 and SK-Mel-103 human melanoma cell lines were obtained from Memorial Sloan Kettering Cancer Center and cultured in DMEM supplemented with $10 \%$ fetal calf serum, $2 \mathrm{mM}$ glutamine and penicillin-streptomycin antibiotics. Cells were kept at $37^{\circ} \mathrm{C}$ under an atmosphere of $5 \%$ carbon dioxide.

Antibodies and other reagents. Mycophenolic acid and guanosine were purchased from Sigma-Aldrich (St. Louis, MO, USA). Angustmycin A was purchased from both Santa Cruz Biotechnology (Santa Cruz, CA, USA) and Enzo Life Sciences (Farmingdale, NY, USA). Mycophenolate Mofetil was purchased from Sigma-Aldrich. The following antibodies were used: mouse monoclonal to GMPS for immunoblotting (Santa Cruz Biotechnology); mouse monoclonal to GAPDH (Ambion, Austin, TX, USA).

Immunoblotting. Whole cell extracts were prepared in NP-40 buffer $(20 \mathrm{mM}$ Tris $\mathrm{pH} 7.4,150 \mathrm{mM} \mathrm{NaCl}$; $\% \mathrm{NP}-40,20 \mathrm{mM} \mathrm{NaF}$ ) supplemented with $1 \mathrm{mM} \mathrm{Na}$ orthovanadate, $1 \mathrm{mM}$ PMSF, and proteases inhibitors (aprotinin $1 \mu \mathrm{g} / \mathrm{ml}$, pepstatin A $1 \mu \mathrm{g} / \mathrm{ml}$, and leupeptin $2 \mu \mathrm{g} / \mathrm{ml})$. Samples (20-80 $\mu \mathrm{g} / \mathrm{lane})$ were resolved on denaturing polyacrylamides gel and transferred onto nitrocellulose membranes. Membranes were incubated $\mathrm{o} / \mathrm{n}$ at $4^{\circ} \mathrm{C}$ with primary antibodies, followed by $1 \mathrm{~h}$ incubation at RT with the appropriate HRP-conjugated secondary antibody. Signals were visualized using the Pierce ECL western blotting substrate and X-ray films (Thermo Scientific, Rockford, IL, USA).

Immunohistochemistry. Formalin-fixed and paraffin-embedded human melanocytic cells, cutaneous and metastatic melanoma tissues were processed at the Pathology Core Facility (Roswell Park Cancer Institute). Positive and negative control slides were supplied by the Pathology Core Facility and were included with every immunochemistry run. The GMPS antibody (ABCAM, Cambridge, MA, USA, Cat\#56530) was visualized with the Novocastra (Newcastle, UK) PowerVision kit, followed by Fast Red (Thermo Scientific). The slides were manually counterstained with hematoxylin. Human tissue specimens were scored for intensity of staining by a board-certified pathologist as described in Wawrzyniak et al. ${ }^{8}$

Plasmids and Infection. Lentiviral vectors encoding short-harpins RNA (shRNA) to GMPS along with a non-silencing control vector were purchased from Sigma-Aldrich (shGMPS \#1 TRCN0000045942; shGMPS \#2 TRCN0000045941). Lentiviral infection protocol was described previously. ${ }^{50,51}$ All infected cells were briefly selected for resistance to puromycin and used in the described assays.

Matrigel-based invasion assay. Invasion assay was performed using the BioCoat Matrigel invasion chambers (BD Bioscience, San Diego, CA, USA) according to the manufacturer's instructions, as described in Wawrzyniak et al. ${ }^{8}$ Experiments were performed in duplicates and repeated at least twice.

Combined gelatin degradation assay. Coverslips were coated with warm Oregon Green 488-conjugated gelatin (Invitrogen, Carlsbard, CA, USA) as described in Wawrzyniak et al..$^{8}$ Melanoma cells $\left(7.5 \times 10^{4}\right)$ were seeded on the coverslips and after $16-\mathrm{h}$ incubation at $37^{\circ} \mathrm{C}$ they were fixed in $4 \%$ paraformaldehyde in PBS. After permeabilization in $0.05 \%$ Triton X-100 in PBS, cells were stained with rhodamine-conjugated phalloidin (Invitrogen, to visualize actin) and hoechst (Thermo Scientific, to visualize nuclei). Coverslips were mounted 
onto glass slides with aqua-mount media (Polysciences, Warrington, PA, USA). Fluorescent images were captured using a Nikon TE2000-E inverted microscope (Melville, NY, USA) equipped with Roper CoolSnap HQ CCD camera and MetaVue software (Molecular Devices, Sunnyvale, CA, USA).

Animal studies using a subcutaneous xenograft model. SK-Mel103 or SK-Mel-28 cells expressing control vector or shRNA to GMPS were inoculated subcutaneously in both flanks of 4- to 6-week-old female SCID mice, which are bred and maintained by the in-house transgenic mouse facility at RPC $\left(1.0 \times 10^{6}\right.$ cells/flank, and $5.0 \times 10^{6}$ cells/flank, respectively). For all cohorts, the time of the appearance of tumor $\geq 2 \mathrm{~mm}$ in at least one dimension was recorded and tumors were measured thereafter every other day. For drug treatment studies, uninfected SK-Mel-103 or SK-Mel-28 cells were inoculated as described above. When tumors reached $100 \mathrm{~mm}^{3}$ in size, mice were randomly assigned to different treatment groups and treated with daily i.p. injection of angustmycin A $(120 \mathrm{mg} / \mathrm{kg}$ in a solution of $10 \%$ DMSO in PBS), MMF ( $30 \mathrm{mg} / \mathrm{kg}$ in a suspension of $0.9 \%$ benzyl alcohol, $0.9 \%$ sodium chloride, $0.5 \%$ carboxymethylcellulose, and $0.4 \%$ polysorbate 80 in water), or the correspondent vehicle control.

Statistical analysis. Student's $t$-Test was used to assess the significance of differences in data obtained in cell-based experiments or tumor xenograft growth assay.

\section{Conflict of Interest}

The authors declare no conflict of interest.

Acknowledgements. We are grateful to $\mathrm{Dr}$ Dominic Smiraglia (RPCl) for critical reading of the manuscript, and to the Pathology Resource Network and the Clinical Data Network at Roswell Park Cancer Institute for providing human specimens. This work has been supported by NIH grants CA120244 (MAN) and CA083081 (DSS), American Cancer Society grant RSG-10-121-01 (MAN), Jennifer Linscott Tietgen Foundation (MAN) and Ruth L Kirschstein National Research Service Award F32CA189622 (AB-S).

1. Neri B, Vannozzi L, Fulignati C, Pantaleo P, Pantalone D, Paoletti C et al. Long-term survival in metastatic melanoma patients treated with sequential biochemotherapy: report of a Phase II study. Cancer Invest 2006; 24: 474-478.

2. Miller AJ, Mihm MC Jr. Melanoma. N Engl J Med 2006; 355: 51-65.

3. Kolli-Bouhafs K, Sick E, Noulet F, Gies JP, De Mey J, Ronde P. FAK competes for Src to promote migration against invasion in melanoma cells. Cell Death Dis 2014; 5: e1379.

4. Dang CV. Links between metabolism and cancer. Genes Dev 2012; 26: 877-890.

5. Tong $X$, Zhao $F$, Thompson $C B$. The molecular determinants of de novo nucleotide biosynthesis in cancer cells. Curr Opin Genet Dev 2009; 19: 32-37.

6. Liu YC, Li F, Handler J, Huang CR, Xiang Y, Neretti N et al. Global regulation of nucleotide biosynthetic genes by c-Myc. PLOS ONE 2008; 3: e2722.

7. Mannava S, Grachtchouk V, Wheeler LJ, Im M, Zhuang D, Slavina EG et al. Direct role of nucleotide metabolism in C-MYC-dependent proliferation of melanoma cells. Cell Cycle 2008; 7: 2392-2400

8. Wawrzyniak JA, Bianchi-Smiraglia A, Bshara W, Mannava S, Ackroyd J, Bagatis A et al. A purine nucloetide biosynthesis enzyme guanosine monophosphate reductase is a suppressor of melanoma invasion. Cell Rep 2013; 5: 493-507.

9. Tedeschi PM, Markert EK, Gounder M, Lin H, Dvorzhinski D, Dolfi SC et al. Contribution of serine, folate and glycine metabolism to the ATP, NADPH and purine requirements of cancer cells. Cell Death Dis 2013; 4: e877.

10. Sievers TM, Rossi SJ, Ghobrial RM, Arriola E, Nishimura P, Kawano M et al. Mycophenolate mofetil. Pharmacotherapy 1997; 17: 1178-1197.

11. Sweeney MJ. Mycophenolic acid and its mechanism of action in cancer and psoriasis. Jpn J Antibiot 1977; 30: 85-92

12. Ransom JT. Mechanism of action of mycophenolate mofetil. Ther Drug Monit 1995; 17 : 681-684.

13. Huh KH, Cho Y, Kim BS, Do JH, Park YJ, Joo DJ et al. The role of thioredoxin 1 in the mycophenolic acid-induced apoptosis of insulin-producing cells. Cell Death Dis 2013; 4: e721.

14. Koehl GE, Wagner F, Stoeltzing $O$, Lang $S A$, Steinbauer $M$, Schlitt $H J$ et al. Mycophenolate mofetil inhibits tumor growth and angiogenesis in vitro but has variable antitumor effects in vivo, possibly related to bioavailability. Transplantation 2007; 83: $607-614$.

15. Rodriguez-Pascual J, Sha P, Garcia-Garcia E, Rajeshkumar NV, De Vicente E, Quijano Y et al. A preclinical and clinical study of mycophenolate mofetil in pancreatic cancer. Invest New Drugs 2013; 31: 14-19.
16. Hirst M, Haliday E, Nakamura J, Lou L. Human GMP synthetase. Protein purification, cloning, and functional expression of cDNA. J Biol Chem 1994; 269: 23830-23837.

17. Zalkin H. GMP synthetase. Methods Enzymol 1985; 113: 273-278.

18. Long H, Cameron S, Yu L, Rao Y. De novo GMP synthesis is required for axon guidance in Drosophila. Genetics 2006; 172: 1633-1642.

19. Rodriguez-Suarez R, Xu D, Veillette K, Davison J, Sillaots S, Kauffman S et al. Mechanismof-action determination of GMP synthase inhibitors and target validation in Candida albicans and Aspergillus fumigatus. Chem Biol 2007; 14: 1163-1175.

20. Tojo S, Hirooka K, Fujita Y. Expression of kinA and kinB of Bacillus subtilis, necessary for sporulation initiation, is under positive stringent transcription control. J Bacteriol 2013; 195: 1656-1665.

21. Faesen AC, Dirac AM, Shanmugham A, Ovaa H, Perrakis A, Sixma TK. Mechanism of USP7/HAUSP activation by its C-terminal ubiquitin-like domain and allosteric regulation by GMP-synthetase. Mol Cell 2011; 44: 147-159.

22. Reddy BA, van der Knaap JA, Bot AG, Mohd-Sarip A, Dekkers DH, Timmermans MA et al. Nucleotide biosynthetic enzyme GMP synthase is a TRIM21-controlled relay of p53 stabilization. Mol Cell 2014; 53: 458-470.

23. van der Knaap JA, Kozhevnikova E, Langenberg K, Moshkin YM, Verrijzer CP. Biosynthetic enzyme GMP synthetase cooperates with ubiquitin-specific protease 7 in transcriptional regulation of ecdysteroid target genes. Mol Cell Biol 2010; 30: 736-744.

24. van der Knaap JA, Kumar BR, Moshkin YM, Langenberg K, Krijgsveld J, Heck AJ et al. GMP synthetase stimulates histone H2B deubiquitylation by the epigenetic silencer USP7. Mol Cell 2005; 17: 695-707.

25. Vasantha N, Freese E. Enzyme changes during Bacillus subtilis sporulation caused by deprivation of guanine nucleotides. J Bacteriol 1980; 144: 1119-1125.

26. Ikehara K, Okamoto M, Sugae K. Induction of Bacillus subtilis sporulation by decoyinine and the concomitant disappearance of ppGpp in vegetative cells. J Biochem 1982; 91 : 1089-1092.

27. Mitani T, Heinze JE, Freese E. Induction of sporulation in Bacillus subtilis by decoyinine or hadacidin. Biochem Biophys Res Commun 1977; 77: 1118-1125.

28. Nicholson WL, Chambliss GH. Effect of decoyinine on the regulation of alpha-amylase synthesis in Bacillus subtilis. J Bacteriol 1987; 169: 5867-5869.

29. Uratani B, Lopez JM, Freese E. Effect of decoyinine on peptidoglycan synthesis and turnover in Bacillus subtilis. J Bacteriol 1983; 154: 261-268.

30. Hotary KB, Allen ED, Brooks PC, Datta NS, Long MW, Weiss SJ. Membrane type I matrix metalloproteinase usurps tumor growth control imposed by the three-dimensional extracellular matrix. Cell 2003; 114: 33-45.

31. Yamaki $\mathrm{H}$, Tanaka N, Umezawa $\mathrm{H}$. Effects of several tumor-inhibitory antibiotics on immunological responses. J Antibiot (Tokyo) 1969; 22: 315-321.

32. Yuntsen H, Ohkuma K, Ishii Y, Yonehara H. Studies on angustmycin. III. J Antibiot (Tokyo) 1956; 9: 195-201.

33. Jayaram HN, Cooney DA, Grusch M, Krupitza G. Consequences of IMP dehydrogenase inhibition, and its relationship to cancer and apoptosis. Curr Med Chem 1999; 6: 561-574.

34. Nagai M, Natsumeda $Y$, Weber G. Proliferation-linked regulation of type II IMP dehydrogenase gene in human normal lymphocytes and HL-60 leukemic cells. Cancer Res 1992; 52: 258-261.

35. Allison AC, Eugui EM. Immunosuppressive and other effects of mycophenolic acid and an ester prodrug, mycophenolate mofetil. Immunol Rev 1993; 136: 5-28.

36. Bentley R. Mycophenolic Acid: a one hundred year odyssey from antibiotic to immunosuppressant. Chem Rev 2000; 100: 3801-3826.

37. Mitsui A, Suzuki S. Immunosuppressive effect of mycophenolic acid. J Antibiot (Tokyo) 1969; 22: $358-363$

38. Lee WA, Gu L, Miksztal AR, Chu N, Leung K, Nelson PH. Bioavailability improvement of mycophenolic acid through amino ester derivatization. Pharm Res 1990; 7 : 161-166.

39. Domhan S, Muschal S, Schwager C, Morath C, Wirkner U, Ansorge W et al. Molecular mechanisms of the antiangiogenic and antitumor effects of mycophenolic acid. Mol Cancer Therap 2008; 7: 1656-1668.

40. Dun $\mathrm{B}, \mathrm{Xu} \mathrm{H}$, Sharma A, Liu H, Yu H, Yi B et al. Delineation of biological and molecular mechanisms underlying the diverse anticancer activities of mycophenolic acid. Int J Clin Exp Pathol 2013; 6: 2880-2886.

41. Siegel R, Ma J, Zou Z, Jemal A. Cancer statistics, 2014. Cancer J Clin 2014; 64: 9-29.

42. Vorsmann H, Groeber F, Walles H, Busch S, Beissert S, Walczak H et al. Development of a human three-dimensional organotypic skin-melanoma spheroid model for in vitro drug testing. Cell Death Dis 2013; 4: e719.

43. DeSantis CE, Lin CC, Mariotto AB, Siegel RL, Stein KD, Kramer JL et al. Cancer treatment and survivorship statistics, 2014. Cancer J Clin 2014; 64: 252-271.

44. Marino ML, Fais S, Djavaheri-Mergny M, Villa A, Meschini S, Lozupone F et al. Proton pump inhibition induces autophagy as a survival mechanism following oxidative stress in human melanoma cells. Cell Death Dis 2010; 1: e87.

45. Romano S, Staibano S, Greco A, Brunetti A, Nappo G, llardi G et al. FK506 binding protein 51 positively regulates melanoma stemness and metastatic potential. Cell Death Dis 2013; 4: $e 578$

46. Hu W, Jin L, Jiang CC, Long GV, Scolyer RA, Wu $Q$ et al. AEBP1 upregulation confers acquired resistance to BRAF (V600E) inhibition in melanoma. Cell Death Dis 2013; 4: e914. 
47. Wansleben S, Davis E, Peres J, Prince S. A novel role for the anti-senescence factor TBX2 in DNA repair and cisplatin resistance. Cell Death Dis 2013; 4: e846.

48. Shtivelman E, Davies MQ, Hwu P, Yang J, Lotem M, Oren M et al. Pathways and therapeutic targets in melanoma. Oncotarget 2014; 5: 1701-1752.

49. Tronnier M, Mitteldorf $\mathrm{C}$. Treating advanced melanoma: current insights and opportunities. Cancer Manag Res 2014; 6: 349-356.
50. Mannava S, Moparthy KC, Wheeler LJ, Natarajan V, Zucker SN, Fink EE et al. Depletion of deoxyribonucleotide pools is an endogenous source of DNA damage in cells undergoing oncogene-induced senescence. Am J Pathol 2013; 182: 142-151.

51. Mannava S, Omilian AR, Wawrzyniak JA, Fink EE, Zhuang D, Miecznikowski JC et al. PP2AB56alpha controls oncogene-induced senescence in normal and tumor human melanocytic cells. Oncogene 2012; 31: 1484-1492.

Supplementary Information accompanies this paper on Cell Death and Differentiation website (http://www.nature.com/cdd) 